\title{
HYPERSPACES OF CONES AND FANS
}

\author{
CARL EBERHART AND SAM B. NADLER, JR. ${ }^{1}$
}

\begin{abstract}
In this paper we investigate the structure of the hyperspaces of subcontinua of some nonlocally connected continua. It is shown that if $X$ is either the cone over any infinite compact metric space or a fan with an infinite number of endpoints, then the space of subcontinua containing the vertex of $X$ is homeomorphic to the Hilbert cube. The hyperspace of subcontinua of a smooth fan (i.e., a subcontinuum of the cone over the Cantor set) is completely described. Also we discuss the question of when two "nicely embedded" copies of the Hilbert cube in a hyperspace have a Hilbert cube sum. In connection with this we describe two spaces, one decomposable and one indecomposable, whose hyperspaces of continua are homeomorphic.
\end{abstract}

1. Introduction. Let $X$ be a metric continuum and let $\mathcal{C}(X)$ be the hyperspace of all subcontinua of $X$ topologized by the Hausdorff metric. Curtis and Schori have proved that if $X$ is locally connected and contains no free arc, then $\mathcal{C}(X)$ is homeomorphic with the Hilbert cube $Q$ [5]. The structure of $\mathcal{C}(X)$ when $X$ is nonlocally connected is not known except in a few specific cases (for a discussion see [14]).

Recently Toruńczyk has given a short proof of the Curtis-Schori theorem as an application of his characterization of $Q$-manifolds [16]. In [9], Torunczyk's characterization was used to show that intervals of continua, $C(A, X)$, in $\mathcal{C}(X)$ are often homeomorphic with $Q$ (note: $\mathcal{C}(A, X)$ denotes the subspace of $\mathcal{C}(X)$ consisting of all $B \in \mathcal{C}(X)$ which contain $A)$. For example if $X$ is the Cantor fan (i.e., the cone over the Cantor set) and $v$ is the vertex of $X$, then $\mathcal{C}(\{v\}, X) \approx Q$ ( $\approx$ means is homeomorphic with). More generally, the following result was shown in [9].

1.1. [9] Assume $Y$ is a compact metric space such that the identity map on $Y$ can be approximated (in the sup metric) by maps $f: Y \rightarrow Y$ such that $f(Y) \neq Y$. Then $C(\{v\}, X) \approx Q$, where $X$ is the cone over $Y$ and $v$ is the vertex of $X$.

In the next section we will improve 1.1 (see Theorem 2.2) and prove an analogue for fans (see Theorem 2.3). In $\$ 3$ we combine 2.3 with a theorem of Anderson ([1] or [2]), 1.3 below, to construct a simple geometric model for the full hyperspace $\mathcal{C}(X)$ of any smooth fan $X$. As a corollary to the construction

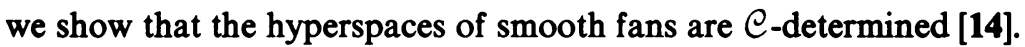

Received by the editors September 14, 1978.

AMS (MOS) subject classifications (1970). Primary 57B20, 54F15.

Key words and phrases. Hyperspace, cone, fan, Hilbert cube, $Z$-set.

${ }^{1}$ The second author was partially supported by National Research Council (Canada) grant no. A5616. 
In $§ 4$, we state and discuss two Hilbert cube sum questions in hyperspaces. Also we give examples of two spaces, one indecomposable and one decomposable, which have homeomorphic hyperspaces.

The principal facts about the Hilbert cube which we make use of are listed here for convenience. A closed subset $A$ of $X$ is called a $Z$-set in $X$ provided the identity map on $X$ can be approximated by maps $f: X \rightarrow X$ such that $f(X) \cap A=\varnothing$.

1.2 (TORUŃCZYK'S CHARACTERIZATION OF $Q$ [16]). If $X$ is a compact metric absolute retract for which the identity map on $X$ can be approximated by maps whose ranges are $Z$-sets in $X$, then $X \approx Q$.

1.3 (ANDERSON'S HOMOGENEITY THEOREM [1]). If $h: A \rightarrow B$ is a homeomorphism between $Z$-sets in $Q$, then $h$ extends to a homeomorphism of $Q$ onto $Q$.

1.4 (HANDEL'S SUM THEOREM [12]). If $A \approx B \approx Q \approx A \cap B$ and $A \cap B$ is a $Z$-set in $A$, then $A \cup B \approx Q$.

2. Vertex intervals. We regard the cone over a compact metric space $Y$ as the quotient space $X=Y \times[0,1] / Y \times\{1\}$. The vertex of $X$ is the point $v=Y \times\{1\}$. For $0<t<1, X$, will be the closed neighborhood $Y \times$ $[t, 1] / Y \times\{1\}$ of $v$ in $X$. In 2.2 below we extend 1.1 to full generality; however, first we need a lemma.

2.1. LemMa. Assume $Y$ is a compact metric space with an infinite number of points. Then either $Y$ satisfies the hypothesis of 1.1 or $Y$ contains a proper closed subset which intersects each component of $Y$.

Proof. Assume $Y$ does not satisfy the hypothesis of 1.1. We will show that for some $\varepsilon>0$ only finitely many components have diameter less than $\varepsilon$. If no such $\varepsilon$ exists, then by the compactness of $Y$, there is a sequence of distinct components of $Y$ converging to a singleton $\left\{y_{0}\right\}$. Now let $\varepsilon>0$ be given and choose two distinct components $C_{1}$ and $C_{2}$ of $Y$ which lie in the $\varepsilon$-neighborhood $N_{e}\left(y_{0}\right)$ of $y_{0}$. Now choose an open and closed set $U$ containing $C_{1} \cup C_{2}$ and contained in $N_{e}\left(y_{0}\right)$, and let $f: Y \rightarrow Y$ be the retraction collapsing $U$ to a point and leaving each point outside $U$ fixed. Since $f(Y) \neq Y$ and $f$ moves no point as much as $2 \varepsilon, Y$ satisfies the hypothesis of 1.1 , contrary to assumption. Hence we assume $\varepsilon>0$ has been chosen so that only finitely many components of $Y$ have diameter less than $\varepsilon$. Denote these components by $C_{1}$, $C_{2}, \ldots, C_{n}$, and choose points $p_{i} \in C_{i}$ for $i=1,2, \ldots, n$. If $Y=\cup_{i=1}^{n} C_{i}$, the set $\left\{p_{1}, p_{2}, \ldots, p_{n}\right\}$ is a proper closed subset ( $Y$ is infinite) which meets each component of $Y$. If $Y \neq \cup \cup_{i=1}^{n} C_{i}$, choose $p \in Y \backslash \cup{ }_{i=1}^{n} C_{i}$ and choose $\delta>0$ so that $\delta<\varepsilon / 2$ and $N_{\delta}(p) \subseteq\left(Y \backslash \cup{ }_{i=1}^{n} C_{i}\right)$. Let $C$ be any component of $Y$ which meets $N_{\delta}(p)$. Then $C$ also meets $Y \backslash N_{\delta}(p)$ since diam $C>\varepsilon>$ $\operatorname{diam} N_{\delta}(p)$. Hence $Y \backslash N_{\delta}(p)$ is a proper closed subset of $Y$ which meets each component of $Y$, and the proof of 2.1 is complete. 
2.2. THEOREM. Suppose $Y$ is a compact metric space with an infinite number of points. Then $\mathcal{C}(\{v\}, X) \approx Q$, where $X$ is the cone over $Y$ and $v$ is the vertex of $X$.

Proof. Assume $Y$ does not satisfy the hypothesis of 1.1 . Fix $0<t<1$. We will show that the interval $\mathcal{C}\left(X_{t}, X\right)$ is a $Z$-set in $\mathcal{C}(\{v\}, X)$. Then, since $\mathcal{C}(\{v\}, X)$ is an absolute retract [9] and the map $f_{t}$ from $\mathcal{C}(\{v\}, X)$ to $\mathcal{C}\left(X_{t}, X\right)$ given by $f_{t}(A)=A \cup X_{t}$ approximates the identity on $C(\{v\}, X)$ as $t \rightarrow 1$, it follows from 1.2 that $\mathcal{C}(\{v\}, X) \approx Q$. Choose, by 2.1 , a proper closed subset $K$ of $Y$ which intersects each component of $Y$. For each $s$, $t<s<1$, define a subcontinuum $K_{s}$ of $X$ as follows:

$$
K_{s}=\{v\} \cup(K \times[s, 1)) \cup(Y \times\{s\}) .
$$

Let $\delta>0$ and choose $s$ so close to 1 that diam $X_{s}<\delta$. Define a function $g_{s}$ on $\mathcal{C}(\{v\}, X)$ by

$$
g_{s}(A)=\left(A \cup X_{s}\right) \backslash\left(X_{s} \backslash K_{s}\right) .
$$

It can be shown (see Lemma 3 of [9]) that $g_{s}$ is continuous from $\mathcal{C}(\{v\}, X)$ into $\mathcal{C}(\{x\}, X) \backslash \mathcal{C}\left(X_{t}, X\right)$ and is within $\delta$ of the identity on $\mathcal{C}(\{v\}, X)$. Thus we conclude that $\mathcal{C}\left(X_{t}, X\right)$ is a $Z$-set in $\mathcal{C}(\{v\}, X)$. This completes the proof of 2.2 .

The cone over a compact totally disconnected metric space is a special type of fan. In general, a fan [3] is defined to be a hereditarily unicoherent, arcwise connected metric space which contains exactly one ramification point (the common part of three otherwise disjoint arcs), called the top of $X$. If $x$, $y \in X$, a fan, then $[x, y]$ denotes the unique arc $x$ to $y$ in $X$ (see [3]). An endpoint of a fan $X$ is a point $e \in X$ such that $e$ is not a cutpoint of any arc in $X$. The following is an analogue of 2.2 for fans.

2.3. TheOREM. Let $X$ be a fan with an infinite number of endpoints and let $t$ be the top of $X$. Then $\mathcal{C}(\{t\}, X) \approx Q$.

Proof. J. B. Fugate has shown that for each $\varepsilon>0$ there is a retraction $r_{e}$ : $X \rightarrow X$ such that $d\left(x, r_{e}(x)\right)<\varepsilon$ for all $x \in X$ and $r_{e}(X)$ is a fan with finitely many endpoints [11]. Hence the induced map $\hat{r}_{e}: \mathcal{C}(X) \rightarrow \mathcal{C}(X)$ given by $\hat{r}_{e}(A)=\left\{r_{e}(a) \mid a \in A\right\}$ is within $\varepsilon$ of the identity on $\mathcal{C}(X)$ and takes the interval $\mathcal{C}(\{t\}, X)$ into itself. We will show that $\hat{r}_{e}(\mathcal{C}(\{t\}, X))$ is a $Z$-set in $\mathcal{C}(\{t\}, X)$; the theorem then follows from 1.2 since $\mathcal{C}(\{t\}, X)$ is an absolute retract.

Since $r_{e}(X)$ has only finitely many endpoints, there is an endpoint $e$ of $X$ such that the arc $[t, e]$ from $t$ to $e$ in $X$ intersects $r_{e}(X)$ only at $t$. For each $s \in[t, e]$ with $s \neq t$, define a map $f_{s}$ from $\mathcal{C}(\{t\}, X)$ into $\mathcal{C}(\{t\}, X)$ by $f_{s}(A)=A \cup[t, s]$. Clearly the maps $f_{s}$ approximate the identity on $\mathcal{C}(\{t\}, X)$ as $s \rightarrow t$; further, the range of $f_{s}$ misses $\hat{r}_{e}(e(\{t\}, X))$. Hence $\hat{r}_{e}(e(\{t\}, X))$ is a $Z$-set in $C(\{t\}, X)$ and the proof of 2.3 is complete. 
3. Hyperspaces of smooth fans. A fan $X$ with top $t$ is said to be smooth [3] provided that if $\left\{x_{n}\right\}$ is a sequence in $X$ converging to a point $x \in X$, then the sequence $\left\{\left[t, x_{n}\right]\right\}$ of arcs in $X$ converges in $C(X)$ to the $\operatorname{arc}[t, x]$. For example, the cone over a compact totally disconnected metric space is easily seen to be a smooth fan. Conversely, it is shown in [10] that every smooth fan is homeomorphic with a subcontinuum of the Cantor fan.

In [7, p. 248], Duda has observed that if $X$ is an $n$-odd (i.e. the cone over $n$ points, $2<n<\infty)$ then $\mathcal{C}(X)$ is homeomorphic with an $n$-cell to the surface of which $n$ 2-cells are attached along a standardly embedded $n$-odd. We describe this model for $\mathcal{C}(X)$ more precisely in 3.2 below, where we show that it has a straightforward generalization to the hyperspace of any smooth fan.

To establish some notation, let $X$ be a smooth fan with top $t$ and endpoints $\left\{e_{\alpha} \mid \alpha \in \Lambda\right\}$. It follows from the definition of smoothness that the set

$$
N[\mathcal{C}(X)]=\{[t, x] \mid x \in X\}
$$

is a homeomorphic copy of $X$ in $C(X)$ (the homeomorphism is the map $x \rightarrow[t, x])$. We call $N[\mathcal{C}(X)]$ the natural part of $\mathcal{C}(X)$. It is also a consequence of the smoothness of $X$ that the set

$$
T[\mathcal{C}(X)]=\bigcup_{\alpha \in \Lambda} \mathcal{C}\left(\left[t, e_{\alpha}\right]\right)
$$

is a closed subspace of $\mathcal{C}(X)$. Furthermore, the hyperspaces $e\left(\left[t, e_{\alpha}\right]\right)$, which are 2-cells, are pairwise disjoint except for $\{t\}$, which they all have in common. We call $T[\mathcal{C}(X)]$ the closure of the two-dimensional part of $\mathcal{C}(X)$. (Compare with Duda [6, p. 279].) We have the following structure theorem.

3.1. Theorem. Let $X$ be a smooth fan with top $t$ and let $N[\mathcal{C}(X)]$ and $T[\mathcal{C}(X)]$ be as defined above. Then

(1) $\mathcal{C}(X)=\mathcal{C}(\{t\}, X) \cup T[\mathcal{C}(X)]$

(2) $\mathcal{C}(\{t\}, X) \cap T[\mathcal{C}(X)]=N[\mathcal{C}(X)]$ and $N[\mathcal{C}(X)]$ is a Z-set in $\mathcal{C}(\{t\}, X)$

(3) if $X$ has infinitely many (respectively, exactly $n, 2<n<\infty)$ endpoints, then $\mathcal{C}(\{t\}, X) \approx Q$ (respectively $\left.\mathcal{C}(\{t\}, X) \approx I^{n}\right)$;

(4) $T[\mathcal{C}(X)] \approx(X \times[0,1]) /(\{t\} \times[0,1])$, the quotient space obtained from $X \times[0,1]$ by shrinking $\{t\} \times[0,1]$ to a point.

Proof. (1) is clear since each $A \in \mathcal{C}(X)$ either contains $t$ or is contained in some arc $\left[t, e_{\alpha}\right]$. The equality in (2) is obvious. The fact that $N[C(X)]$ is a $Z$-set in $C(\{t\}, X)$ can be seen as follows. Regard $X$ as a subcontinuum of the Cantor fan $C \times[0,1] / C \times\{1\}$, where $C$ is the Cantor set. Then for $0<\varepsilon<$ 1, the map $f_{e}: \mathcal{C}(\{t\}, X) \rightarrow \mathcal{C}(\{t\}, X) \backslash N[\mathcal{C}(X)]$ given by $f_{e}(A)=A \cup\left(C_{1-e}\right.$ $\cap X)$, where $C_{1-\varepsilon}$ is the subcontinuum $C \times[1-\varepsilon, 1] / C \times\{1\}$ of the Cantor fan, approximates the identity on $\mathcal{C}(\{t\}, X)$ as $\varepsilon \rightarrow 1$. This completes the proof of (2).

The infinite case of (3) follows from 2.3; the finite case can be established 
directly by the homeomorphism $h: X_{i=1}^{n}\left[t, e_{i}\right] \rightarrow \mathcal{C}(\{t\}, X)$ defined by $h\left(x_{1}, x_{2}, \ldots, x_{n}\right)=\cup_{i=1}^{n}\left[t, x_{i}\right]$.

Proof of (4). Since $X$ embeds in the Cantor fan, we can regard $X$ as embedded in the place so that $t=(0,0)$ and each of the $\operatorname{arcs}\left[t, e_{\alpha}\right]$ in $X$ is a convex segment. Define a function $f$ from $X \times[0,1]$ to $T[\mathcal{C}(X)]$ by $f(x, s)=$ [sx, x]. It is easily verified that $f$ is a mapping of $X \times[0,1]$ onto $T[\mathcal{C}(X)]$ whose only nondegenerate point inverse is $f^{-1}(\{t\})=\{t\} \times[0,1]$. Hence $T[\mathcal{C}(X)]$ is homeomorphic with the quotient space $X \times[0,1] /\{t\} \times[0,1]$. This completes the argument for 3.1.

In the proof of $(4)$ above, note that $N[\mathcal{C}(X)] \approx X$ is naturally identified with the subspace $\left\{f^{-1}(t)\right\} \cup\{\{(x, 0)\} \mid x \neq t\}$ of the quotient space $P=X$ $\times[0,1] /\{t\} \times[0,1]$. Denote this subspace by $X_{0}$. The next result shows how to realize the hyperspace $\mathcal{C}(X)$ of a smooth fan $X$ by attaching $P$ to a cell (either $Q$ or $I^{n}$ ) along a nicely embedded copy of $X_{0}$. We follow Dugundji's terminology for attaching maps [8].

3.2. TheOREM. Let $X$ be a smooth fan and let $P$ and $X_{0}$ be as above. Then:

(1) If $X$ has an infinite number of endpoints, let $h: X_{0} \rightarrow Q$ be any embedding of $X_{0}$ as a $Z$-set in $Q$. Then the space obtained by attaching $P$ to $Q$ along $X_{0}$ with the attaching map $h$ is homeomorphic with $\mathcal{C}(X)$; that is, $P \cup_{h} Q \approx$ $\mathrm{e}(X)$.

(2) If $X$ has $n$ endpoints, $2<n<\infty$, let $h$ be an embedding of $X_{0}$ into the boundary of $I^{n}\left(\approx S^{n-1}\right)$ which is tame in the sense that there is a homeomorphism of the boundary of $I^{n}$ onto itself taking $h\left(X_{0}\right)$ to an $n$-odd whose arcs $\left[t, e_{i}\right]$ are convex. Then $P \cup_{h} I^{n} \approx \mathcal{C}(X)$.

Proof of (1). Let $f^{*}$ be the homeomorphism of $T[\mathcal{C}(X)]$ onto $P$ induced by the map $f$ defined in the proof of (4) of 3.1. Clearly $f^{*}$ takes $N[\mathcal{C}(X)]$ onto $X_{0}$. Now the composition $h f^{*}$ then takes the $Z$-set $N[\mathcal{C}(X)]$ in the Hilbert cube $\mathcal{C}(\{t\}, X)$ (see 3.1(2) and 2.3) homeomorphically onto the $Z$-set $h\left(X_{0}\right)$ in the Hilbert cube $Q$. Hence, by $1.3, h f^{*}$ extends to a homeomorphism $k: \mathcal{C}(\{t\}, X)$ $\rightarrow Q$. Let $\rho$ be the quotient map from the free union of $P$ and $Q, P+Q$, to $P \cup_{h} Q$. Then it follows using (1) and (2) of 3.1 that the formula

$$
g(A)= \begin{cases}\rho f^{*}(A) & \text { if } A \in T[\mathcal{C}(X)], \\ \rho k(A) & \text { if } A \in \mathcal{C}(\{t\}, X)\end{cases}
$$

defines a homeomorphism from $\mathcal{C}(X)$ onto $P \cup_{h} Q$.

Proof of (2). In $I^{n}=X_{i=1}^{n}[0,1]$, realize $X$ as the union of the edges $E_{i}=\left\{\left(x_{1}, x_{2}, \ldots, x_{n}\right) \mid x_{j}=0\right.$ if $\left.j \neq i, 0<x_{i}<1\right\}$. Then $C(\{t\}, X)$ maps homeomorphically onto $I^{n}$ by the map $P(A)=\left(x_{1}, \ldots, x_{n}\right)$ where $p_{i}\left(A \cap E_{i}\right)$ $=\left[0, x_{i}\right]$ and $p_{i}$ is the projection of $I^{n}$ onto the $i$ th coordinate interval. By hypothesis there exists a homeomorphism $k$ from the boundary of $I^{n}$ onto itself which takes $p(X)$ onto $h\left(X_{0}\right)$. Extend $k$ to a homeomorphism $k^{*}$ from $I^{n}$ onto itself (this is clearly possible). As in the proof of (1), let $f^{*}$ be the homeomorphism from $T[\mathcal{C}(X)]$ onto $P$ and let $\rho: P+I^{n} \rightarrow P \cup_{h} I^{n}$ be the 
quotient map. Use (1) and (2) of 3.1 to deduce that the formula

$$
g(A)= \begin{cases}\rho f^{*}(A) & \text { if } A \in T[\mathcal{C}(X)], \\ \rho k^{*}(A) & \text { if } A \in \mathcal{C}(\{t\}, X)\end{cases}
$$

defines a homeomorphism of $\mathcal{C}(X)$ onto $P \cup_{h} I^{n}$. This completes the proof of 3.2.

It is interesting to note that, in the proof of 3.2 , more care must be taken with the attaching homeomorphism $h$ in the finite case than in the infinite case. This is because it is possible to embed the arc in nonequivalent ways into the boundary of $I^{n}$ as long as $3<n<\infty$. Such nonequivalent embeddings are not possible in the case $n=3$ because of the Schoenflies theorem and in the infinite case because of Anderson's Theorem 1.3.

With the aid of 3.2 , we can construct a particularly simple geometric model for $\mathcal{C}(X), X$ a smooth fan, we describe when $X$ has an infinite number of endpoints. Regard $X$ as embedded in the Hilbert cube $Q=\times_{i=1}^{\infty}[0,1]$ so that the projection $p_{i}$ of $X$ onto the $i$ th coordinate interval is $\{0\}$ for $i>2$. Further assume that each of the arcs $\left[t, e_{\alpha}\right]$ in $X$ is convex (i.e., $s x \in[t, x]$ for $0<s<1$ and $x \in X)$ and has length $<1$. For each $A \in T[\mathcal{C}(X)]$, define $M(A)$ to be the point of $A$ such that $A \subset[t, M(A)]$ and $l(A)=\inf \{d(t, x) \mid x$ $\in A\}$. It is readily seen that $M$ and $l$ are continuous functions which together separate the points of $T[\mathcal{C}(X)]$. Hence the map $h: T[\mathcal{C}(X)] \rightarrow[0,1] \times Q$ given by $h(A)=(l(A), M(A))$ is an embeddng which takes $N[\mathcal{C}(X)]$ onto $\{0\} \times X$ which is a $Z$-set in $\{0\} \times Q$. So the subset $h(T[\mathcal{C}(X)]) \cup\{0\} \times Q$ is homeomorphic with $\mathcal{C}(X)$, by 3.2(1). (See Figure 1.)

We conclude our study of the hyperspace of a smooth fan with the following corollary. In the terminology of $[14,0.61]$ it says that the members of the class of smooth fans are $\mathcal{C}$-determined. Thus it provides information concerning a question in $[14,0.62]$.

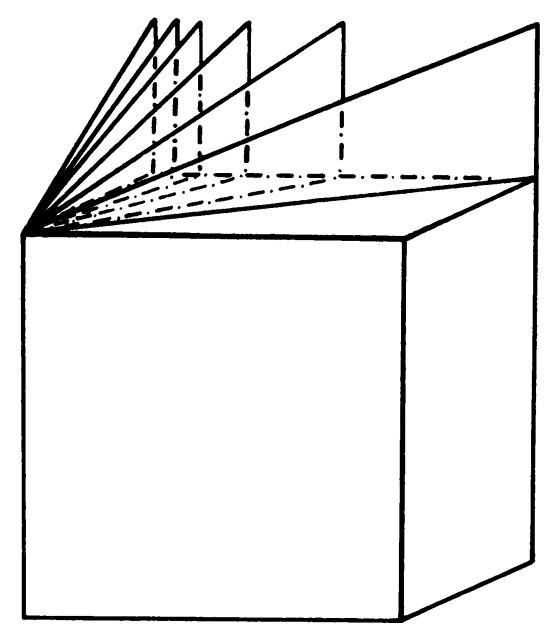

FIGURE 1 
3.3. Corollary. If $X_{1}$ and $X_{2}$ are smooth fans with $\mathcal{C}\left(X_{1}\right) \approx \mathcal{C}\left(X_{2}\right)$, then $X_{1} \approx X_{2}$

Proof. Let $t_{1}$ and $t_{2}$ denote the tops of $X_{1}$ and $X_{2}$, respectively, and let $h$ : $\mathcal{C}\left(X_{1}\right) \rightarrow \mathcal{C}\left(X_{2}\right)$ be a homeomorphism. It follows from 3.1 that $h\left(\mathcal{C}\left(\left\{t_{1}\right\}, X_{1}\right)\right)$ $=\mathcal{C}\left(\left\{t_{2}\right\}, X_{2}\right)$ and $h\left(T\left[\mathcal{C}\left(X_{1}\right)\right]\right)=T\left[\mathcal{C}\left(X_{2}\right)\right]$. Hence by (2) of 3.1, $h\left(N\left[\mathcal{C}\left(X_{1}\right)\right]\right)=N\left[\mathcal{C}\left(X_{2}\right)\right]$. Therefore since $N\left[\mathcal{C}\left(X_{i}\right)\right] \approx X_{i}, i=1,2, X_{1} \approx X_{2}$. This proves 3.3.

It would be interesting to know if the class of fans is $\mathcal{C}$-determined.

4. Two Hilbert cube sum questions for hyperspaces. In [1], Anderson conjectured that if $Q_{1}, Q_{2}$ and $Q_{1} \cap Q_{2}$ are Hilbert cubes, then $Q_{1} \cup Q_{2}$ is a Hilbert cube. He verified this for the case when $Q_{1} \cap Q_{2}$ is a $Z$-set in each of $Q_{1}$ and $Q_{2}$ [1]. Handel has shown that $Q_{1} \cap Q_{2}$ need only be a $Z$-set in $Q_{1}$ (see 1.4), and Sher's example [15] indicates that Handel's result may be the best possible in the general setting. In this section we will inquire into the extent to which Anderson's conjecture holds when $Q_{1}$ and $Q_{2}$ are embedded nicely in some hyperspace. Specifically we ask the following two questions.

4.1. Question. Let $p, q \in X$, a metric continuum, and assume that $\mathcal{C}(\{p\}, X)$, $\mathcal{C}(\{q\}, X)$ and $\mathcal{C}(\{p\}, X) \cap \mathcal{C}(\{q\}, X)$ are Hilbert cubes. Is $\mathcal{C}(\{p\}, X) \cup$ $\mathcal{C}(\{q\}, X)$ a Hilbert cube?

We call a closed subset $\mathbb{Q}$ of $\mathcal{C}(X)$ convex in $\mathcal{C}(X)$ if $A, B \in \mathbb{Q}$ and $A \subseteq C \subseteq B, C \in \mathcal{C}(X)$ implies that $C \in \mathbb{Q}$. Intervals of continua are special types of convex sets, and 4.1 can be asked more generally:

4.2. Question. Assume $\mathcal{Q}$ and $\mathscr{B}$ are convex sets in $\mathcal{C}(X)$ such that $\mathscr{Q}, \mathscr{B}$ and $\mathcal{Q} \cap \mathscr{B}$ are Hilbert cubes. Then is $\mathbb{Q} \cup \mathscr{B}$ a Hilbert cube?

The answer to 4.1 in the case when $X$ is a Peano continuum which contains no local cutpoints is yes and can be proved using a lemma of Curtis [4].

4.3. TheOREM. Let $X$ be a Peano continuum which contains no local cutpoint. Then if $p, q \in X, \mathcal{C}(\{p\}, X) \cup \mathcal{C}(\{q\}, X)$ is a Hilbert cube.

Proof. Note first that $X$ does not contain a free arc and we know that $\mathcal{C}(\{p\}, X)$ and $\mathcal{C}(\{q\}, X)$ are Hilbert cubes from [5] or [9]. The fact that $\mathcal{C}(\{p\}, X) \cap \mathcal{C}(\{q\}, X)$ is a Hilbert cube can be shown using the argument in Theorem 4 of [9]. Then we apply Lemma 2.1 of [4] to conclude that $\mathcal{C}(\{p\}, X) \cap \mathcal{C}(\{q\}, X)$ is a $Z$-set in $\mathcal{C}(\{p\}, X)$, and so by Handel's sum theorem 1.4, $\mathcal{C}(\{p\}, X) \cup \mathcal{C}(\{q\}, X)$ is a Hilbert cube.

To illustrate that 4.1 and 4.2 can hold for $X$ nonlocally connected, we describe two examples.

4.4. Example. Let $X_{1}$ and $X_{2}$ be copies of the Cantor fan with vertices $p$ and $q$ respectively constructed in the plane so that $p$ is an endpoint of $X_{2}, q$ is an endpoint of $X_{1}$ and $X_{1} \cap X_{2}$ is an arc $[p, q]$ with endpoints $p$ and $q$ (see Figure 2). 


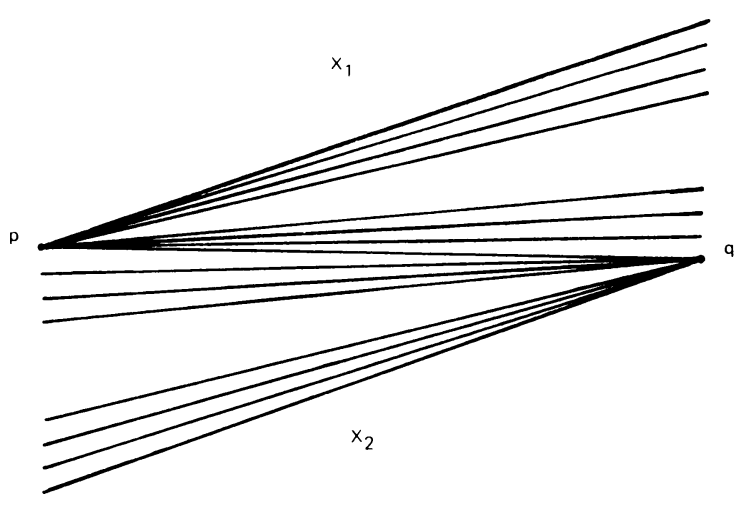

FIgURE 2

Thus the space $X=X_{1} \cup X_{2}$ is a dendroid which is not locally connected at any point. It is readily verified using 6 of $[9]$ that $C(\{p\}, X)$ and $C(\{q\}, X)$ are both Hilbert cubes. Then, using the fact that $\mathcal{C}([p, q], X)$ is homeomorphic with the interval of continua containing the point $[p, q]$ in the quotient space $X /[p, q]$, it is easy to see that $\mathcal{C}(\{p\}, X) \cap \mathcal{C}(\{q\}, X)$, which is $\mathcal{C}([p, q], X)$, is a Hilbert cube. We will show that $\mathcal{C}(\{p\}, X) \cup \mathcal{C}(\{q\}, X)$ is a Hilbert cube. To see this let $\mathcal{Q}$ be the closure of $\mathcal{C}(\{p\}, X) \backslash \mathcal{C}(\{q\}, X)$ in $\mathcal{C}(X)$. Clearly $\mathcal{Q}=\mathcal{C}\left(\{p\}, X_{1}\right) \approx Q$. Also $\mathbb{Q} \cap \mathcal{C}(\{q\}, X)=\mathcal{C}\left([p, q], X_{1}\right) \approx$ $Q$. To complete the argument we need only show, by 1.4 , that $\mathscr{Q} \cap \mathcal{C}(\{q\}, X)$ is a $Z$-set in $\mathcal{C}(\{q\}, X)$. So for each $\varepsilon>0$, choose an arc $A_{e}$ in $X_{2} \backslash[p, q)$ of diameter less than $\varepsilon$ and having $q$ as one endpoint. Then the maps $f_{\varepsilon}$ : $\mathcal{C}(\{q\}, X) \rightarrow \mathcal{C}(\{q\}, X)$ defined by $f_{\varepsilon}(A)=A \cup A_{\varepsilon}$ demonstrate that $\mathbb{Q} \cap$ $\mathcal{C}(\{q\}, X)$ is a $Z$-set in $\mathcal{C}(\{q\}, X)$. (Note: $\mathcal{Q} \cap \mathcal{C}(\{q\}, X)$ is also a $Z$-set in $\mathcal{C}\left(\{p\}, X_{1}\right)$; however, $\mathcal{C}(\{p\}, X) \cap \mathcal{C}(\{q\}, X)$ is not a $Z$-set in either $\mathcal{C}(\{p\}, X)$ or $\mathcal{C}(\{q\}, X)$ since its interior in each is nonvoid.)

We remark that it is possible to construct, using the above observations and techniques analogous to those in $\$ 3$, a geometric model for the full hyperspace $C(X)$ of the space $X$ in 4.4 .

A topological property is said to be $C$-determined if whenever $X$ has the property and $\mathcal{C}(X) \simeq \mathcal{C}(Y)$, then $Y$ has the property. For example, local connectedness is $C$-determined as is hereditary indecomposability $[14,1.61]$.

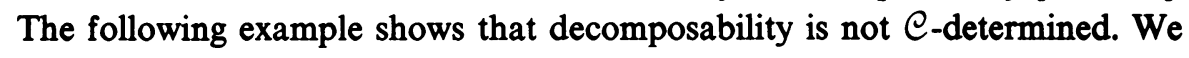
include the example here since the verifications in the example are related to 4.2.

4.5. EXAMPLE. Let $Y$ denote the indecomposable continuum as described in [13, p. 205]. We modify $Y$ in the following way. Let $A=[a, b]$ be an arc in $Y$ with endpoints $a$ and $b$, and let $\left\{x_{n}\right\}$ be a monotone sequence in $[a, b]$ converging to $a$. Let $X_{1}$ be the quotient space obtained from $Y$ by identifying all the odd terms of $\left\{x_{n}\right\}$ with $a$ and sewing each arc $\left[x_{2 n+1}, x_{2 n+2}\right]$ to the arc $\left[x_{2 n+2}, x_{2 n+3}\right]$ in reverse order. Let $X_{2}$ be the unit disk and let $X$ be the space 
obtained by attaching a boundary point of $X_{2}$ to the point $a$ of $X_{1}$. (See Figure 3.)

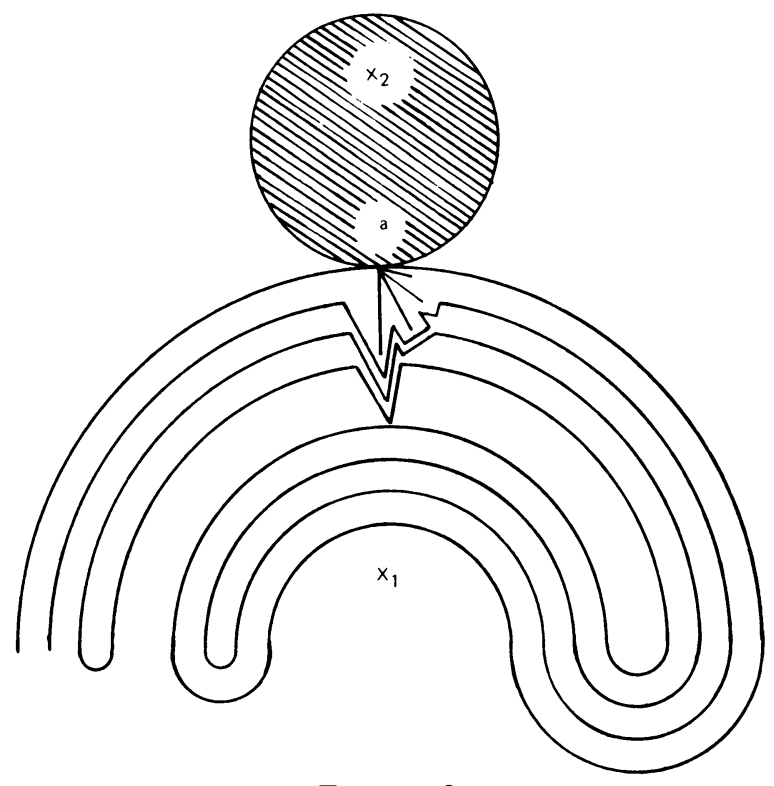

FIGURE 3

Clearly the continuum $X_{1}$ is indecomposable while the continuum $X$ is decomposable. We will show that $\mathcal{C}(X) \approx \mathcal{C}\left(X_{1}\right)$.

First we note that $\mathcal{C}(X)$ decomposes into $\mathcal{C}\left(X_{1}\right) \cup \mathcal{C}(\{a\}, X) \cup \mathcal{C}\left(X_{2}\right)$ with $\mathcal{C}(\{a\}, X)$ intersecting $\mathcal{C}\left(X_{1}\right)$ and $\mathcal{C}\left(X_{2}\right)$ in $\mathcal{C}\left(\{a\}, X_{1}\right)$ and $\mathcal{C}\left(\{a\}, X_{2}\right)$, respectively. Now $\mathcal{C}\left(X_{2}\right) \approx Q$ by [5] and $\mathcal{C}(\{a\}, X) \approx Q$ by [9]. Also $\mathcal{C}\left(X_{2}\right)$ $\cap \mathcal{C}(\{a\}, X)=\mathcal{C}\left(\{a\}, X_{2}\right) \approx Q$ by [9], and using the maps $f_{n}: \mathcal{C}(\{a\}, X) \rightarrow$ $\mathcal{C}(\{a\}, X) \backslash \mathcal{C}\left(\{a\}, X_{2}\right)$ given by $f_{n}(A)=A \cup\left[x_{2 n+1}, x_{2 n+2}\right]$ we see that $\mathcal{C}\left(\{a\}, X_{2}\right)$ is a $Z$-set in $\mathcal{C}(\{a\}, X)$. Hence by $1.4 \mathcal{C}(\{a\}, X) \cup \mathcal{C}\left(X_{2}\right) \approx Q$.

For each $n$, let $f_{n}: X_{1} \rightarrow X_{1}$ be a retraction such that the arc $\left[x_{2 n+1}, x_{2 n+2}\right]$ meets $f_{n}\left(X_{1}\right)$ only in the point $x_{2 n+1}=a$. Clearly we can choose the maps $f_{n}$ so that $f_{n} \rightarrow 1_{X_{1}}$ as $n \rightarrow \infty$. Thus the induced maps $f_{n}^{*}: \mathcal{C}\left(\{a\}, X_{1}\right) \rightarrow$ $\mathcal{C}\left(\{a\}, X_{1}\right)$ are $Z$-maps which approach the identity on $\mathcal{C}\left(\{a\}, X_{1}\right)$. Hence by $1.2 \mathcal{C}\left(\{a\}, X_{1}\right) \approx Q$. In addition it can be seen that the sets $f_{n}^{*}\left(\mathcal{C}\left(\{a\}, X_{1}\right)\right)$ lie in the interior of $\mathcal{C}\left(\{a\}, X_{1}\right)$ relative to $\mathcal{C}\left(X_{1}\right)$. Hence the boundary $\mathscr{B}$ of $\mathcal{C}\left(\{a\}, X_{1}\right)$ in $\mathcal{C}\left(X_{1}\right)$ is a $Z$-set in $e\left(\{a\}, X_{1}\right)$. But also $\mathscr{B}$ is a $Z$-set in $\mathcal{C}(\{a\}, X) \cup \mathcal{C}\left(X_{2}\right)$. To see this, extend $f_{n}$ to $g_{n}: X \rightarrow X$ by defining $g_{n}(x)=x$ if $x \in X_{2}$ and note that the induced map $g_{n}^{*}: \mathcal{C}(\{a\}, X) \cup \mathcal{C}\left(X_{2}\right) \rightarrow$ $\mathcal{C}(\{a\}, X) \cup \mathcal{C}\left(X_{2}\right)$ approaches the identity on $\mathcal{C}(\{a\}, X) \cup \mathcal{C}\left(X_{2}\right)$ as $n \rightarrow$ $\infty$, and that im $g_{n}^{*} \cap \mathscr{B} \neq \varnothing$.

Now by 1.3 , there is a homeomorphism $h$ from $\mathcal{C}(\{a\}, X) \cup \mathcal{C}\left(X_{2}\right)$ onto $\mathcal{C}\left(\{a\}, X_{1}\right)$ which is the identity on $\mathscr{B}$. Extend $h$ by the identity to all of $\mathcal{C}(X)$. This completes the discussion of 4.5 . 


\section{REFERENCES}

1. R. D. Anderson, Topological properties of the Hilbert cube and the infinite product of open intervals, Trans. Amer. Math. Soc. 126 (1967), 220-216.

2. T. A. Chapman, Lectures on Hilbert cube manifolds, CBMS, Regional Conference Series in Math., no. 28, Amer. Math. Soc., Providence, R. I., 1975.

3. J. J. Charatonik, On fans, Dissertationes Math. 54 (1967).

4. D. W. Curtis, Hyperspaces of noncompact metric spaces (preprint).

5. D. W. Curtis and R. M. Schori, Hyperspaces of Peano continua are Hilbert cubes, Fund. Math. (to appear).

6. R. Duda, On the hyperspace of subcontinua of a finite graph. I, Fund. Math. 62 (1968), 265-286.

7. __ On the hyperspace of subcontinua of a finite graph. II, Fund. Math. 63 (1968), 225-255.

8. James Dugundji, Topology, Allyn and Bacon, Boston, Mass., 1966.

9. Carl Eberhart, Intervals of continua which are Hilbert cubes, Proc. Amer. Math. Soc. 68 (1978), 220-224.

10. _ A note on smooth fans, Colloq. Math. 20 (1969), 89-90.

11. J. B. Fugate, Retracting fans onto finite fans, Fund. Math. 71 (1971), 114-125.

12. Mike Handel, On certain sums of Hilbert cubes, General Topology and Appl. 9 (1978), 19-28.

13. K. Kuratowski, Topology, Vol. 1, Academic Press, New York, 1966.

14. S. B. Nadler, Jr., Hyperspaces of sets $-A$ text with research questions, Pure and Applied Math. Series, vol. 49, Marcel Dekker, New York, 1978.

15. R. Sher, The union of two Hilbert cubes meeting in a Hilbert cube need not be a Hilbert cube, Proc. Amer. Math. Soc. 63 (1977), 150-152.

16. H. Torunczyk, On CE-images of the Hilbert cube and characterizations of Q-manifolds (preprint).

Department of Mathematics, Universtty OF Kentucky, LeXINGton, Kentucky 40506 